\title{
Single-Stage Dental Implant Placement with Indirect Sinus Floor Elevation Technique: A Clinical Report
}

\section{Smita P Vaidya, Arun N Khalikar, Shankar P Dange, Priyanka Ganesh Govindwar}

\begin{abstract}
The posterior maxilla is a challenging site for dental implant rehabilitation. Anatomic limitations in this region provide challenges that may affect successful osseointegration and the fabrication of a functional and esthetic implant-supported prosthesis. The technique of sinus floor elevation has expanded prosthetic options by enabling the placement of additional implant support in maxillary segments with atrophic ridges and pneumatized sinuses.
\end{abstract}

Keywords: Indirect sinus lift, Osteotome, Implant in atrophic maxilla.

How to cite this article: Vaidya SP, Khalikar AN, Dange SP, Govindwar PG. Single-Stage Dental Implant Placement with Indirect Sinus Floor Elevation Technique: A Clinical Report. Int J Oral Implantol Clin Res 2012;3(3):150-153.

\section{Source of support: Nil}

Conflict of interest: None declared

\section{INTRODUCTION}

Implant insertion into the posterior maxilla is more demanding owing to compromised bone quality and quantity. ${ }^{1-5}$ The presence of the maxillary sinus floor limits the available bone height for implant placement. Different methods, such as tilted implants, short implants, vertical bone augmentation and sinus floor elevation, have been used to overcome these problems. Sinus floor elevation can be performed through a lateral window ${ }^{6-8}$ or via a crestal access. ${ }^{9-14}$ The most commonly used technique is sinus floor elevation through a lateral window which was first presented by Tatum in 1977 and was first published by Boyne and James in $1980{ }^{6,14-16}$ However, this bone augmentation procedure is considered to be invasive, time-consuming and expensive. ${ }^{8,17,18}$ Compared to minimally invasive methods, the major shortcomings of this method are potential nerve and vascular injury, requirement of good surgical skills and patient discomfort. Lateral bone fenestration has limitations similar to hinge osteotomy. Summers ${ }^{9}$ in 1994 introduced a less aggressive procedure for sinus floor elevation with immediate implant placement known as osteotomy sinus floor elevation (OSFE). A crestal approach rather than lateral window was used followed by osteome for elevation of sinus membrane and floor and simultaneously implant was placed. Simultaneously, some kind of graft may or may not be placed. The procedure is less invasive as compared to lateral window technique, requires less time, less traumatic, minimal damage to vital adjacent structure and the postoperative morbidity is less. Recent studies have shown that the prognosis of implants placed by OSFE was same as that of conventional technique. ${ }^{15}$ This case report illustrates sinus lift with OSFE procedure with bone grafting followed by implant placement.

\section{CASE REPORT}

A 22-year-old patient was reported to the Department of Prosthodontics with missing maxillary left first molar (Fig. 1). Suggested treatment as approved by the patient consisted of sinus floor elevation using a crestal approach with sinus lift osteotomes along with graft material and simultaneous implant placement. Step-by-step procedure is as follows:

a. Preoperative evaluation: Bone width and height were measured on computed tomographic (CT) scan. Radiographic stent was fabricated from diagnostic wax up and used as surgical stent also.

b. Surgical phase:

1. Antibiotic prophylaxis was initiated a day before surgery.

2. Under local anesthesia, crestal approach was used.

3. Soft tissue punch was made with the help of a punch guide.

4. Initially, a round burr was used to open a defect through the marginal cortical bone. Increasing diameter of burrs was used to enlarge the site and

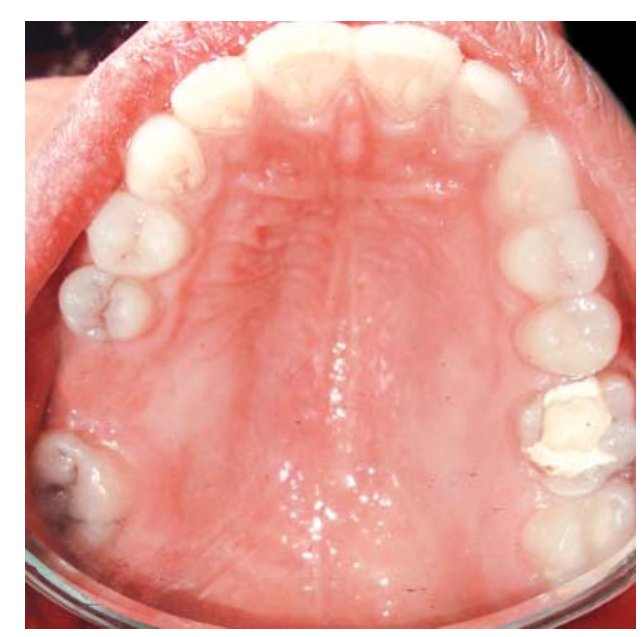

Fig. 1: Preoperative photograph 
preparation of surgical site was assessed at regular interval with radiovisiography.

5. Drilling up to $1 \mathrm{~mm}$ away from the floor was continued with the 2.1, 2.8, 3.3 and $3.65 \mathrm{~mm}$ drills until final preparation.

6. Then expansion osteotomes were used.

7. Light tapping with a mallet carefully collapsed the sinus floor into the sinus cavity elevating the Schneiderian membrane.

8. Prepared bone graft material with beta-tricalcium phosphate and demineralized freeze-dried bone, which acts as a shock absorber, was added to the preparation site with a carrier. Elevation of the maxillary sinus membrane was achieved using the \#3 osteotome that was used previously to force the graft ahead of its tip to achieve the sinus floor up fracture.

9. Integrity of the sinus membrane was confirmed by the Valsalva maneuver.

10. Implant of dimension $(4.2 \times 11.5 \mathrm{~mm})$ was placed. Primary stability was assessed by finger pressure

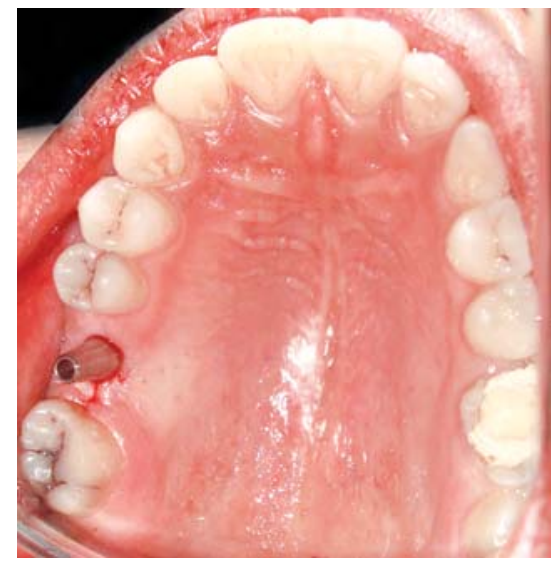

Fig. 2: Straight abutment placement

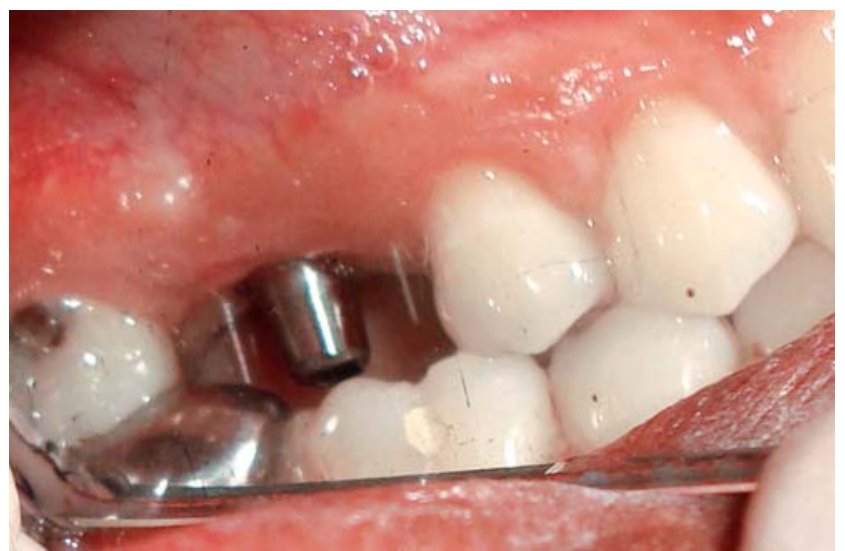

Fig. 3: Occlusal relation of abutment exerted on the implant. The implant showed primary stability. Stability can also be increased by the threads or by placing the implant deeper.

11. Abutment was then positioned over the implant and occlusal height adjusted (Figs 2 and 3). Implant was loaded with temporary restoration.

12. Postoperatively (Fig. 4), patient was instructed to rinse the mouth twice a day with a $0.12 \%$ chlorhexidine solution for 2 weeks after surgery. Antibiotics were prescribed for 7 days.

13. After a mean healing period of 7 months, patient was rehabilitated with fixed crown (Fig. 5).

\section{DISCUSSION}

Elevation of the maxillary sinus floor was first reported by Boyne in the1960s. Fifteen years later, Boyne and James reported elevation of the maxillary sinus floor in patients with large, pneumatized sinus cavities in preparation for the placement of blade implants. It is evident that the reduced vertical bone height in the posterior maxillary region often

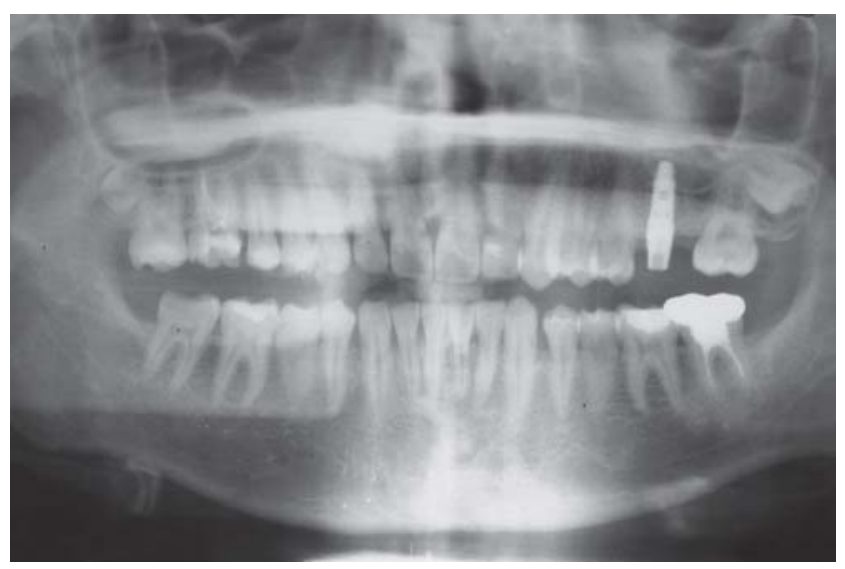

Fig. 4: Radiograph showing sinus lift

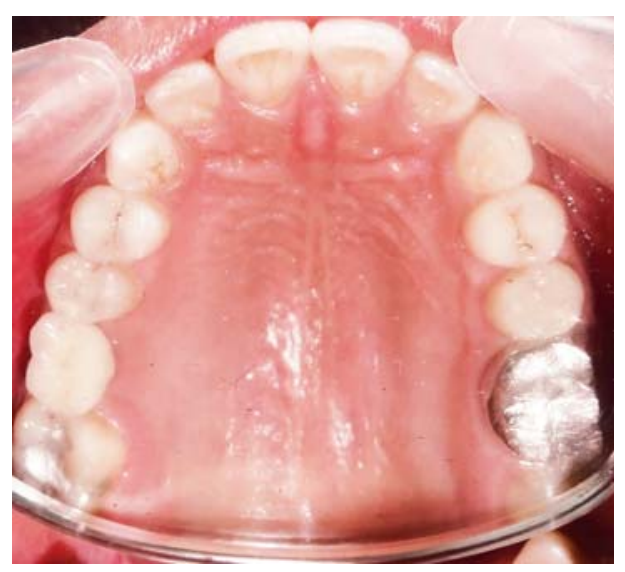

Fig. 5: Completed restoration 
limited standard implant placement. Elevation of the maxillary sinus floor is an option in solving this problem. Various surgical techniques have been presented to enter the sinus cavity elevating the sinus membrane and placing bone grafts. To date, two main approaches to the maxillary sinus floor elevation procedure can be found in the literature. The first approach, lateral antrostomy, is the classic and the more commonly performed technique originally described by Tatum. More recently, Summers advocated a second approach: The crestal approach, using osteotomes. The crestal approach is more conservative method. In 2003, Wallace and Froum published a systematic review on the effect of maxillary sinus floor elevation and the survival of dental implants. The criteria for review included human studies with a minimum of 20 interventions, a follow-up time of 1 year of functional loading and with the outcome variable of implant survival being reported. Recently, Piezo instruments have been used to open the window of the lateral wall but the risk of perforation when the membrane was elevated from the sinus floor remains.

In this case we used the osteotome sinus floor elevation procedure described by Summers which involves a grafting material that is condensed in the osteotomy site to elevate the sinus membrane. The advantages of this procedure were the avoidance of invasive surgery and permitting treatment within a single surgical step. To enhance the primary stability in low-density bone, the use of osteotomes is more relevant than the use of drills. By compression, the osteotomes can laterally condense bone, thus creating a denser interface at the placed implants, ${ }^{21}$ improving the initial bone to implant contact. ${ }^{22}$

The complication can occur if the Schneiderian membrane is perforated, the filling material can migrate into the sinus and lead to inflammation. ${ }^{19,20}$ Proper patient evaluation and surgical site preparation will help to avoid this complication.

\section{SUMMARY}

Implant rehabilitation of edentulous atrophied posterior maxilla can be greatly extended and simplified using implants the OSFE technique. The procedure is short, less invasive with minimal chances of postoperative complication and appears to be predictable. It also allows treating the compromised posterior maxilla with reliable long-term results.

\section{REFERENCES}

1. Adell R, Lekholm U, Rockler B, Branemark PI. A 15-year study of osseointegrated implants in the treatment of the edentulous jaw. Int J Oral Surg 1981;10:387-416.
2. Bryant SR. The effects of age, jaw site, and bone condition on oral implant outcomes. Int J Prosthodont 1998;11:470-90.

3. Esposito M, Hirsch JM, Lekholm U, Thomsen P. Biological factors contributing to failures of osseointegrated oral implants. (I). Success criteria and epidemiology. Eur J Oral Sci 1998;106:527-51.

4. Sennerby L, Roos J. Surgical determinants of clinical success of osseointegrated oral implants: A review of the literature. Int J Prosthodont 1998;11:408-20.

5. Truhlar RS, Orenstein IH, Morris HF, Ochi S. Distribution of bone quality in patients receiving endosseous dental implants. J Oral Maxillofac Surg 1997;55:38-45.

6. Boyne PJ, James RA. Grafting of the maxillary sinus floor with autogenous marrow and bone. J Oral Surg 1980;38:613-16.

7. Hatano N, Shimizu Y, Ooya K. A clinical long-term radiographic evaluation of graft height changes after maxillary sinus floor augmentation with a 2:1 autogenous bone/xenograft mixture and simultaneous placement of dental implants. Clin Oral Implants Res 2004;15:339-45.

8. Jensen OT, Shulman LB, Block MS, Iacono VJ. Report of the Sinus Consensus Conference of 1996. Int J Oral Maxillofac Implants 1998;13(Suppl):11-45.

9. Summers RB. The osteotome technique: Part 3-less invasive methods of elevating the sinus floor. Compendium 1994;15: 698-702.

10. Summers RB. A new concept in maxillary implant surgery: The osteotome technique. Compendium 1994;15:152-58.

11. Bragger U, Gerber C, Joss A, Haenni S, Meier A, Hashorva E, et al. Patterns of tissue remodeling after placement of ITI dental implants using an osteotome technique: A longitudinal radiographic case cohort study. Clin Oral Implants Res 2004;15:158-66.

12. Cavicchia F, Bravi F, Petrelli G. Localized augmentation of the maxillary sinus floor through a coronal approach for the placement of implants. Int J Periodontics Restorative Dent 2001;21:475-85.

13. Rosen PS, Summers R, Mellado JR, Salkin LM, Shanaman RH, Marks $\mathrm{MH}$, et al. The bone-added osteotome sinus floor elevation technique: Multicenter retrospective report of consecutively treated patients. Int J Oral Maxillofac Implants 1999;14:853-58.

14. Tatum H Jr. Maxillary and sinus implant reconstructions. Dent Clin North Am 1986;30:207-29.

15. Emmerich D, Att W, Stappert C. Sinus floor elevation using osteotomes: A systematic review and meta-analysis. J Periodontol 2005;76:1237-51.

16. Smiler DG, Johnson PW, Lozada JL, Misch C, Rosenlicht JL, Tatum OH Jr, et al. Sinus lift grafts and endosseous implants: Treatment of the atrophic posterior maxilla. Dent Clin North Am 1992;36:151-88.

17. Shulman LB, Jensen OT. Sinus Graft Consensus Conference. Introduction. Int J Oral Maxillofac Implants 1998;13(Suppl):5-6.

18. Nedir R, Bischof M, Vazquez L, Szmukler-Moncler S, Bernard JP. Osteotome sinus floor elevation without grafting material: A 1-year prospective pilot study with ITI implants. Clin Oral Implants Res 2006;17:679-86.

19. Pikos MA. Maxillary sinus membrane repair: Update on technique for large and compete perforations. Implant Dent 2008;17:24-31.

20. Raghoebar GM, Timmenga NM, Reintsema H, Stegenga B, Vissink A. Maxillary bone grafting for insertion of endosseous implants: Results after 12 to 124 months. Clin Oral Implants Res 2001;12:279-86. 
21. Hahn J. Clinical uses of osteotomes. J Oral Implantol 1999;25:23-29.

22. Nkenke E, Radespiel-Troger M, Wiltfang J, Schultze-Mosgau S, Winkler G, Neukam FW. Morbidity of harvesting of retromolar bone grafts: A prospective study. Clin Oral Implants Res 2002 Oct;13(5):514-21.

\section{ABOUT THE AUTHORS}

\section{Smita P Vaidya (Corresponding Author)}

Associate Professor, Department of Prosthodontics, Government Dental College and Hospital, Aurangabad, Maharashtra, India, e-mail: smitakhalikar@yahoo.com

\section{Arun N Khalikar}

Associate Professor, Department of Prosthodontics, Government Dental College and Hospital, Nagpur, Maharashtra, India

\section{Shankar P Dange}

Professor, Department of Prosthodontics, Government Dental College and Hospital, Aurangabad, Maharashtra, India

\section{Priyanka Ganesh Govindwar}

Postgraduate Student, Department of Prosthodontics, Government Dental College and Hospital, Aurangabad, Maharashtra, India 